\title{
Short term treatment results of local ablation with water-cooled microwave antenna for liver cancer: Comparison with radiofrequency ablation
}

\author{
KANEHIKO SUWA ${ }^{1,2}$, TOSHIHITO SEKI ${ }^{2}$, RINAKO TSUDA ${ }^{1}$, MASAO YAMASHINA $^{1,2}$, \\ MIKI MURATA $^{1,2}$, TAKASHI YAMAGUCHI ${ }^{1}$, AKIYOSHI NISHIO $^{1}$ and KAZUICHI OKAZAKI ${ }^{1}$ \\ ${ }^{1}$ The Third Department of Internal Medicine, Division of Gastroenterology and Hepatology, \\ Kansai Medical University, Hirakata, Osaka 573-1101; ${ }^{2}$ Kansai Medical University Medical Center, \\ Liver Disease Center, Moriguchi, Osaka 570-8507, Japan
}

Received August 10, 2019; Accepted December 6, 2019

DOI: $10.3892 / \mathrm{mco} .2020 .1983$

\begin{abstract}
The aim of the present study was to evaluate the efficacy and safety of the new-generation percutaneous microwave ablation (MWA) compared with the radiofrequency ablation (RFA) system for the treatment of hepatocellular carcinoma (HCC). A retrospective study was conducted from January 2014 to February 2019. A total of 44 patients and 52 nodules (mean tumor size, $17.2 \pm 4.9 \mathrm{~mm}$ ) were treated with MWA, and 55 patients and 70 nodules (mean tumor size, $17.7 \pm 6.4 \mathrm{~mm}$ ) were treated with RFA. After 4 days of treatment, the direct effects of ablation were assessed using dynamic CT, and after discharge, a follow-up dynamic CT scan was performed every 3-4 months. Treatment efficacy, complications and local recurrence were recorded. For MWA and RFA, the average number of CT sessions were $1.05 \pm 0.23$ and $1.28 \pm 0.54$, respectively, and the mean ablation times were $5.0 \pm 2.0$ and $8.1 \pm 4.8 \mathrm{~min}$. Following MWA and RFA, the ablation ranges that were evaluated with the axial images were $31.9 \pm 5.5$ and $33.3 \pm 9.0 \mathrm{~mm}$, respectively, in the long-axis diameter and $27.6 \pm 5.3$ and $23.4 \pm 6.8 \mathrm{~mm}$, respectively, in the short-axis diameter. The flatness ratios of the ablation regions were $0.13 \pm 0.09$ and $0.29 \pm 0.14$ (axial image) and $0.11 \pm 0.07$ and $0.28 \pm 0.14$ (coronal image), respectively. The rates of complete tumor necrosis were comparable. The complication rates were $13.6 \%$ (MWA) and $14.5 \%$ (RFA), which were not significantly different. The cumulative local recurrence rates were not significantly different between the two methods (one-year recurrence rate, MWA: 6.91\%, RFA: 5.17\%). MWA was therefore indicated to be an effective treatment for HCC
\end{abstract}

Correspondence to: Dr Kanehiko Suwa, The Third Department of Internal Medicine, Division of Gastroenterology and Hepatology, Kansai Medical University, 2-3-1 Shimmachi, Hirakata, Osaka 573-1101, Japan E-mail: suwakan@hirakata.kmu.ac.jp

Key words: hepatocellular carcinoma, microwave ablation, radiofrequency ablation, spherical ablation, local recurrence in respect to session number, treatment time and spherical ablation.

\section{Introduction}

Hepatocellular carcinoma (HCC) is one of the most common causes of cancer-related mortality worldwide and accounts for $5.7 \%$ of new cancer cases. This malignancy tends to occur in livers damaged by chronic infection with hepatitis B or hepatitis $\mathrm{C}$ viruses, alcohol abuse and excessive fat deposits in the context of liver cirrhosis (1-3). In patients with early-stage HCC, while surgical resection is the best definitive treatment for patients with adaptation, percutaneous local treatment (PLT) is the best treatment option for patients with early-stage HCC who are not surgical candidates (4-6). The two most commonly used forms of PLT are radiofrequency ablation (RFA) and microwave ablation (MWA), which are heat-based thermal ablation methods. Because of small size of the necrotic area achieved with one microwave ablation treatment, RFA has been the most frequently used method worldwide and has been shown to be a safe and effective therapeutic option (7-9). However, nodules adjacent to large vessels may often be incompletely ablated due to the heat sink effect. Overall, $10-25 \%$ of HCC patients may not be eligible for RFA (10). The major limitation of conventional MWA systems is the lack of predictability of the ablation zone size and shape. Therefore, a specific new-generation microwave ablation (MWA) system, the Emprint Ablation System (Covidien), which uses an internally water-cooled microwave antenna, was designed to create large predictable spherical zones of ablation that are not impacted by varying tissue environments $(11,12)$. The purpose of this study was to verify the treatment effectiveness and safety of this new MWA system compared with the treatment results obtained with the RFA system and to verify whether this device is effective for the treatment of HCC.

\section{Patients and methods}

Patients. This retrospective study population consisted of 44 patients with 52 nodules treated with MWA between 
July 2017 and February 2019 and 55 patients with 70 nodules treated with RFA between January 2016 and October 2017. To evaluate the efficacy of PLT with regard to local control of the lesions, we excluded patients with extrahepatic metastases or portal tumor thrombosis detected by various imaging modalities, those with a tendency toward severe bleeding, and those whose liver function was Child-Pugh Class $\mathrm{C}$ with refractory ascites. The treatment was limited to a solitary nodular tumor located on one subsegment. The size of each lesion was $<3.0 \mathrm{~cm}$ in diameter. Tumors showing extrahepatic extension and those located near the hilar region were excluded. Informed consent for this procedure was obtained from the patients and his or her family members. Among the 52 nodules treated with MWA, 40 were treated with conventional transcatheter arterial chemoembolization (c-TACE) using iodized oil emulsion (4-6 ml, Lipiodol, Andre Guerbet) before PLT, and of the 70 nodules treated with RFA, 58 were treated with c-TACE before PLT to evaluate the grade of cancer in detail and to visualize the tumor area for easy assessment of the treated margin after treatment. If the clinical diagnosis was difficult, the histological diagnosis of tumors was confirmed by US-guided fine needle biopsy.

The follow-up period ended in February 2019. Table I details the baseline clinical characteristics of the MWA and RFA groups. In both treatment groups, the proportions of men were higher, and the underlying liver disease was most commonly $\mathrm{HCV}$ infection, followed by non-B, non-C patients. Only prothrombin time was significantly lower in the RFA group than in the MWA group, but there were very few differences in the clinical data between the two groups. Additionally, mean tumor diameters and dispersion of locations were not different between the two groups.

The study protocol was conducted with the approval (approval no. 2018143) of the Ethics Committee of Kansai Medical University Medical Center (Moriguchi, Japan). All procedures performed in studies involving human participants were in accordance with the ethical standards of Clinical Research Board of Kansai Medical University Medical Center and with the 1964 Helsinki Declaration and its later amendments or comparable ethical standards. Informed consent was obtained from all individual participants included in the present study.

Equipment. MWA: Emprint ${ }^{\mathrm{TM}}$ Ablation Generator with Thermosphere Technology with Emprint ${ }^{\mathrm{TM}}$ Long Percutaneous Antenna (30 cm; Covidien).

RFA: Cool-tip RF Generator with Cool-tip RF needle (25x3 cm; Covidien).

PLT procedure. After pretreatment with $0.1 \mathrm{mg}$ of fentanyl (Janssen Pharma) and 1.25 mg of droperidol (Daiichi Sankyo), the tumor was detected using an ultrasound diagnostic system (TUS-A300 Aplio300; Canon) with an ultrasound transducer (PVT-382BT; Canon). Subsequently, local anesthesia was administered as $0.5 \%$ lidocaine hydrochloride (Aspen Japan), and a guide needle (MWA, 12Gx140 mm; RFA, 14Gx145 mm; Hakko Medical) was inserted into the vicinity of the tumor under the guidance of ultrasound with a puncture adapter (UAGV-027A; Canon) connected to the transducer. After the inner needle of the guide was removed, the antenna (electrode) was inserted through the outer needle of the guide to place the antenna (electrode) in the tumor area. In the case of MWA, the output energy was gradually increased to $45 \mathrm{~W}$ for $30 \mathrm{sec}$, to $60 \mathrm{~W}$ for $30 \mathrm{sec}$, and then to $75 \mathrm{~W}$ until the end of treatment, whereas in the case of RFA, the output energy was gradually increased to $80 \mathrm{~W}$ for $60 \mathrm{sec}$ and to $100 \mathrm{~W}$ for $60 \mathrm{sec}$, followed by cauterization until the end of treatment at $120 \mathrm{~W}$. Treatments were terminated until the ablation margin was included in the high-echoic area on the ultrasound screen.

Evaluation of treatment effect, shape of necrotic area and follow-up. After 4 days of PLT, the direct effect of ablation was determined by dynamic CT, and the axial and coronal necrotic areas were measured with the long and short diameters perpendicular to it, respectively. Furthermore, we used the equation for the flatness ratio to find out how spherical the necrotic areas were, which is important for predicting treatment range. The flatness ratio of the necrotic area in all patients was calculated as follows: $f=1-b / a$, where $f$ is the flatness ratio, $a$ is the long diameter of the ellipse, and $b$ is the short diameter of the ellipse. The flatness ratio indicates how flat an ellipse is compared with a sphere, and the more spherical the ellipse is, the closer the flatness ratio comes to 0 (Fig. 1A and B). Complete tumor necrosis was defined as $100 \%$ tumor-necrotizing effect, and we defined the treatment margin (TM) as the ablation region being wider across the entire circumference than the low density area in the late phase of pretreatment dynamic CT. In case treated with c-TACE before PLT, TM was defined as wider than the accumulation area of the iodinated oil emulsion over the entire circumference. We subclassified the areas with and without TM (Fig. 1C and D).

After their discharge from the hospital, we closely followed all patients. Dynamic CT scans were performed every 3-4 months. When imaging studies revealed intrahepatic recurrence, the diagnosis was confirmed by $\mathrm{CT}$ angiography and/or US-guided tumor biopsy. Local recurrence was defined as recurrence of nodule in the treatment area or the margin of the treatment area in patients.

Statistical analysis. The local recurrence rate of HCC after PLT was determined using the Kaplan-Meier method. Local recurrence curves were compared between MWA and RFA by means of the log-rank test. The clinical data of the patients undergoing each procedure were compared with the Mann-Whitney U test, while underlying liver diseases and tumor segments were compared with Pearson's Chi-square test. $\mathrm{P}<0.05$ was considered to indicate a statistically significant difference.

\section{Results}

Treatment efficacy. All patients completed PLT in one day. Treatment was terminated once the ablation margin was included in the high-echoic area on the ultrasound screen. With regard to MWA, most of the nodules were treated with only one session (94.2\%), but three nodules required two sessions (mean: $1.05 \pm 0.23$ sessions). However, with regard to RFA, one session was used to treat 53 nodules (75.7\%), two sessions were used to treat 14 nodules $(20 \%)$, and three sessions were used to treat 3 nodules (4.2\%) (mean: 1.28 \pm 0.54 sessions). 
Table I. Baseline clinical data and patient characteristics.

\begin{tabular}{|c|c|c|c|}
\hline Variables & MWA & RFA & P-value \\
\hline Patient (n) & 44 & 55 & \\
\hline Nodule (n) & 52 & 70 & \\
\hline Observation period (days, mean) & $236 \pm 137$ & $602 \pm 252$ & \\
\hline Sex (male/female) & $30 / 14$ & $44 / 11$ & ns \\
\hline Age & $73.4 \pm 7.7$ & $73.2 \pm 8.8$ & ns \\
\hline Background (B/C/NBNC) & $3 / 29 / 12$ & $8 / 31 / 16$ & ns \\
\hline TACE (yes/no) & $40 / 12$ & $58 / 12$ & ns \\
\hline Child-Pugh class (A/B) & $37 / 7$ & $48 / 10$ & ns \\
\hline Prothrombin time $(\%)$ & $84.5 \pm 15.9$ & $78.0 \pm 13.1$ & 0.0238 \\
\hline Albumin $(\mathrm{g} / \mathrm{dl})$ & $3.9 \pm 0.5$ & $3.8 \pm 0.4$ & ns \\
\hline Total bilirubin (mg/dl) & $1.0 \pm 0.5$ & $0.8 \pm 0.3$ & ns \\
\hline Platelet $\left(10^{4} / \mu 1\right)$ & $13.8 \pm 5.8$ & $13.1 \pm 6.8$ & $\mathrm{~ns}$ \\
\hline ALBI & $-2.59 \pm 0.56$ & $-2.52 \pm 0.42$ & ns \\
\hline $\mathrm{AFP}(\mathrm{ng} / \mathrm{ml})$ & $67.2 \pm 233.8$ & $32.8 \pm 111.0$ & ns \\
\hline $\mathrm{DCP}(\mathrm{mAU} / \mathrm{ml})$ & $86.6 \pm 124.1$ & $121.0 \pm 276.6$ & ns \\
\hline Segment (left/medial/anterior/posterior) & $9 / 8 / 19 / 16$ & $7 / 4 / 34 / 25$ & ns \\
\hline Tumor long diameter (mm) & $17.2 \pm 4.9$ & $17.7 \pm 6.4$ & ns \\
\hline Tumor short diameter (mm) & $14.0 \pm 4.9$ & $13.7 \pm 5.4$ & ns \\
\hline
\end{tabular}

MWA, microwave ablation; RFA, radiofrequency ablation; B, hepatitis B virus; $\mathrm{C}$, hepatitis $\mathrm{C}$ virus; NBNC, non-B non-C; TACE, transcatheter arterial chemoembolization; ALBI, albumin-bilirubin-grade; AFP, $\alpha$-fetoprotein; DCP, Des- $\gamma$-carboxy prothrombin.
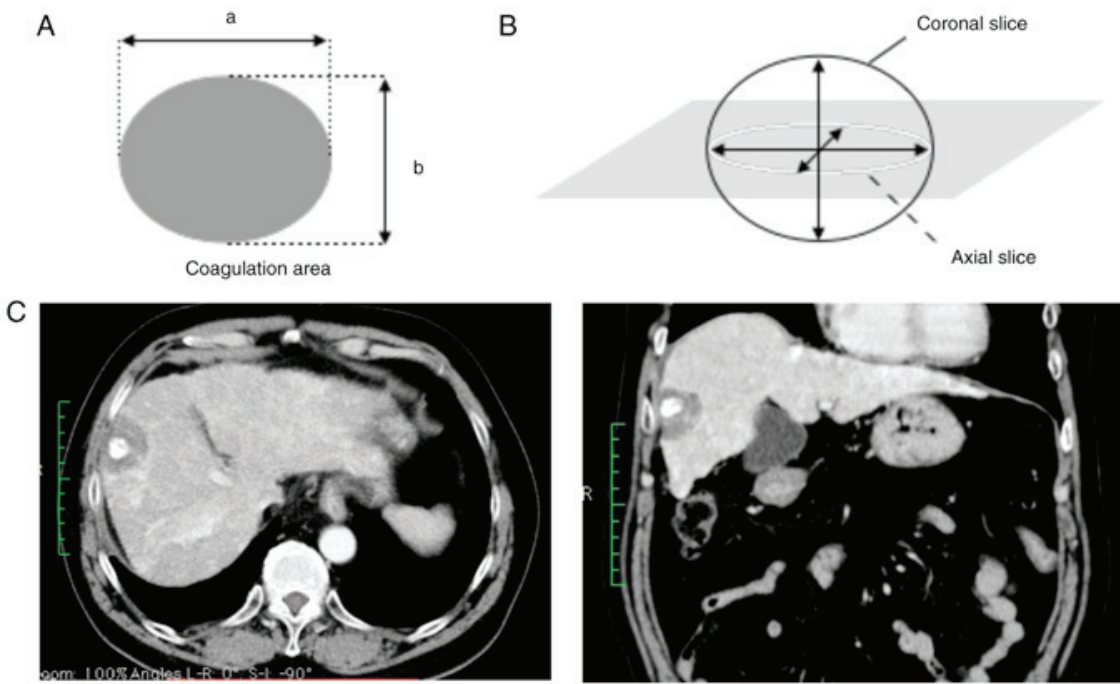

With treatment margin
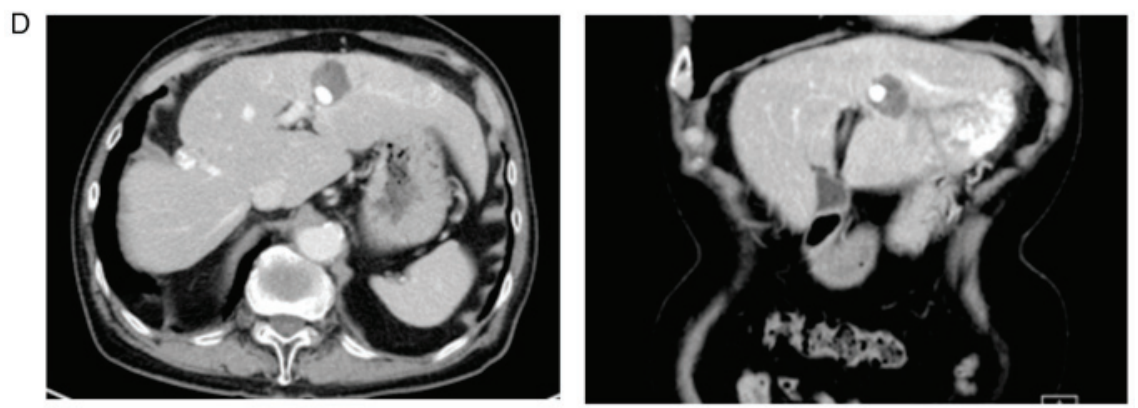

Without treatment margin

Figure 1. Evaluation of the shape of the necrotic area. (A) The long diameter (a) and the short diameter (b) in the maximum necrotic range were measured, and the flatness ratio was defined as $\mathrm{f}=1-\mathrm{b} / \mathrm{a}$. (B) This equation was applied to both axial and coronal slices. (C) The ablation region was wider across the entire circumference than the iodized oil emulsion accumulation area with a treatment margin. (D) Without a treatment margin. 
A
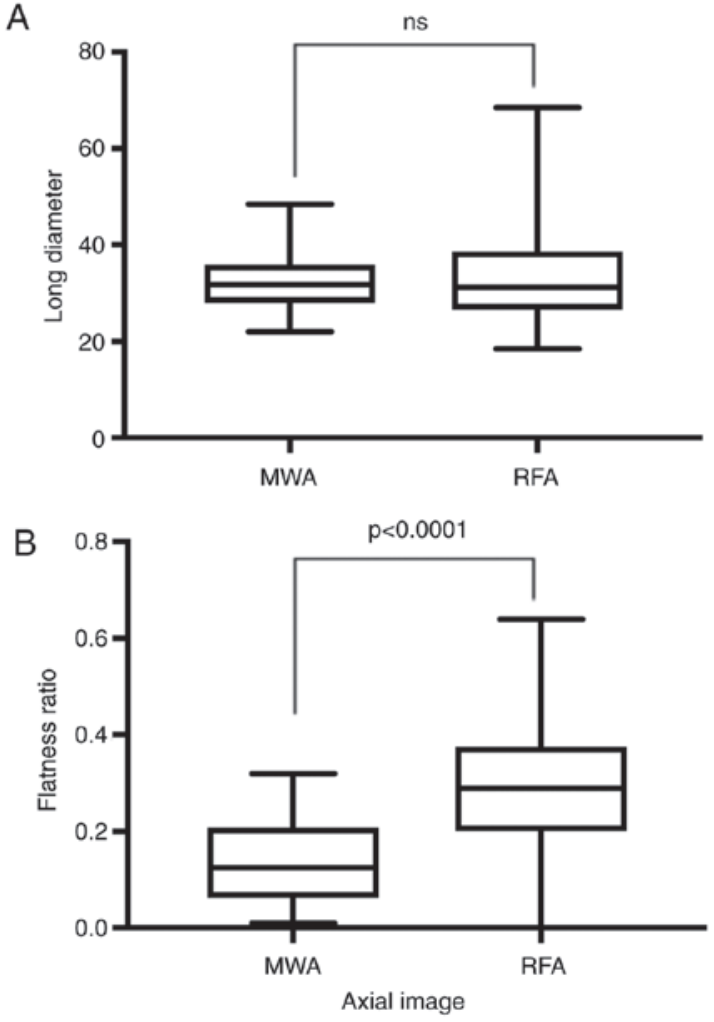
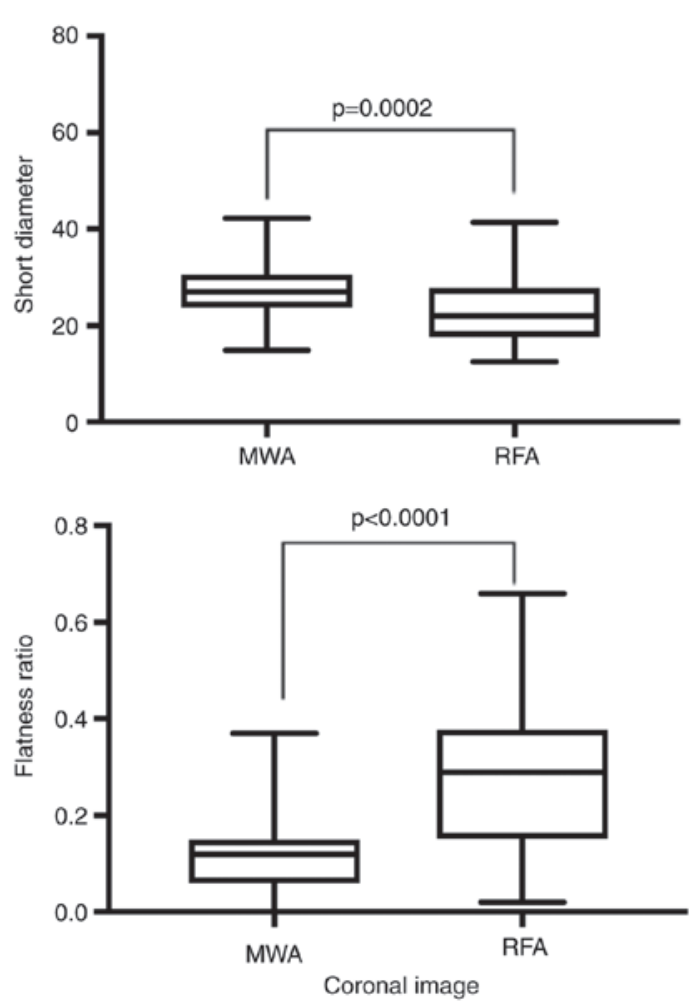

Figure 2. Comparison of the necrotic region between the two methods. (A) In the long diameter, there was no significant difference between MWA and RFA; however, in the short diameter, MWA was able to acquire a larger necrotic area than RFA. (B) The flatness ratio was lower in MWA than in RFA in both axial and coronal images. MWA, microwave ablation; RFA, radiofrequency ablation.

The mean ablation times in MWA and RFA were 5.0 \pm 2.0 and $8.1 \pm 4.8 \mathrm{~min}(\mathrm{P}=0.0066)$, respectively. The ablation ranges evaluated with the axial images for MWA and RFA were $31.9 \pm 5.5$ and $33.3 \pm 9.0 \mathrm{~mm}$, respectively, in the long-axis diameter and $27.6 \pm 5.3$ and $23.4 \pm 6.8 \mathrm{~mm}$, respectively, in the short-axis diameter, and there was a significant difference only in the short-axis diameter $(\mathrm{P}=0.0002)$. The flatness ratios of the ablation region were $0.13 \pm 0.09$ for MWA and $0.29 \pm 0.14$ for RFA on the axial image $(\mathrm{P}<0.0001)$ and $0.11 \pm 0.07$ for MWA and $0.28 \pm 0.14$ for RFA on the coronal image ( $<<0.0001$; Fig. 2). Compared with RFA, MWA caused spherical ablation in a shorter time with fewer punctures. Forty-eight of the 52 nodules treated with MWA showed complete tumor necrosis (with TM 35 nodules: without TM 13 nodules), while sixty-four of the 70 nodules treated with RFA showed complete tumor necrosis (with TM 37 nodules: Without TM 27 nodules). Patients who had insufficient ablation underwent additional PLT or surgery at a later date. Although there was no significant difference in the rate of complete tumor necrosis between the two groups, the treatment margin acquisition rate was somewhat higher for MWA than RFA (MWA/RFA: 72.9/57.8\%) (Table II).

Side effects and complications. With regard to the clinically relevant complications that developed during hospitalization, liver infarction occurred in one patient undergoing MWA and in two patients undergoing RFA. After MWA, three cases of bleeding and subcapsular hematoma occurred; two of the cases spontaneously regained hemostasis due to bed rest, and one case required intravascular embolization. After RFA, three cases of subcapsular hematoma occurred, but all cases achieved spontaneous hemostasis due to bed rest. With regard to other complications, one patient experienced a skin burn during RFA, and one patient experienced a marked decrease in blood pressure due to activation of the vaso-vagal reflex during cauterization by RFA, which necessitated the cessation of ablation. During the observation period, we detected biloma of the treatment area in two patients who had undergone MWA and in one patient who had undergone RFA. There were no statistically significant differences in the incidence rates of complications between the MWA and RFA groups (MWA/RFA: 13.6/14.5\%). None of the patients developed local dissemination of the cancer cells along the puncture line in this study.

Local recurrence. Among the patients who achieved complete tumor necrosis, local recurrence was observed in three patients treated with MWA and ten patients treated with RFA. The cumulative local recurrence rates were not significantly different between the MWA and RFA groups $(\mathrm{HR}=0.82$ (0.18-3.73) log-rank=0.8036, one-year cumulative recurrence rate; MWA/RFA: 6.91/5.17\%) (Fig. 3). Table II shows the results of the subgroup analysis according to the presence of a TM. Although local recurrence did not occur in MWA patients who obtained complete necrosis with TM, a comparison could not be made because the number of cases and observation period were insufficient.

\section{Discussion}

With the positive influence of the surveillance program in high-risk patients, $\mathrm{HCC}$ is being detected at increasingly 
Table II. Local recurrence with and without a TM and time to recurrence.

Days until recurrence

\begin{tabular}{lcccc} 
Variables & Nodules & Local recurrence & Shortest & Longest \\
\cline { 3 - 5 } MWA & & & & - \\
With TM & 35 & 0 & 223 & 238 \\
Without TM & 13 & 2 & & \\
RFA & 37 & 2 & 411 & 453 \\
With TM & 27 & 8 & 229 & 538 \\
Without TM & & & 359
\end{tabular}

TM, treatment margin; MWA, microwave ablation; RFA, radiofrequency ablation.

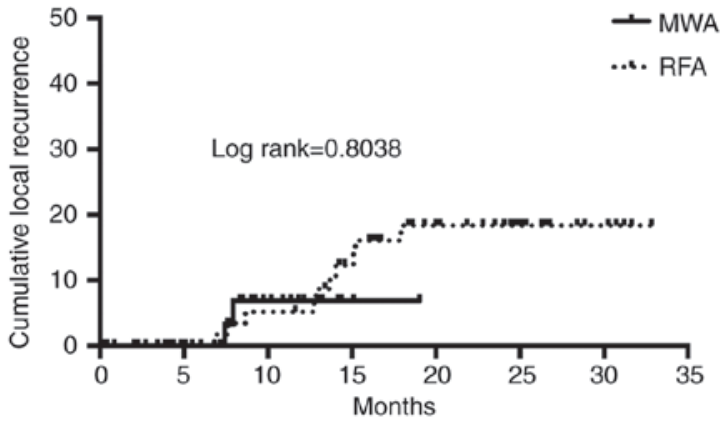

No. at risk

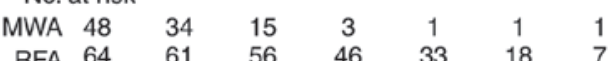

Figure 3 . The cumulative local recurrence rate was not significantly different between MWA and RFA. MWA, microwave ablation; RFA, radiofrequency ablation.

smaller sizes. MWA and RFA have high local tumor control abilities and result in low levels of invasion and are therefore recommended as the most appropriate treatment options of early-HCC $(13,14)$. Previous studies had reported that first-generation MWA is a useful treatment for small HCC tumors $(15,16)$, and in several reports, MWA and RFA have been reported to yield equivalent results in terms of therapeutic effects and complications $(17,18)$. However, compared with RFA, first-generation MWA has a narrow range of ablation in one session; therefore, more treatment sessions are needed to obtain complete tumor necrosis with the appropriate margin. Under these circumstances, RFA has been reported to be equivalent to surgery in terms of the survival and tumor control rates $(19,20)$, and it has been adopted as the most popular thermal ablation method.

However, RFA has a disadvantage, namely, if the target lesion is in the vicinity of a large vessel, RFA cannot sufficiently cauterize the tissue due to the heat sink effect; this is a phenomenon that occurs when thermal energy diffuses away from the target lesion due to blood flow in adjacent vessels (10). MWA is less susceptible to the heat sink effect because of its higher temperatures and shorter ablation times $(21,22)$. This reduced susceptibility is ultimately due to differences in the mechanisms of action between MWA and RFA. RFA uses current, whereas MWA uses electromagnetic energy (23). The limitations of first-generation MWA were the unpredictable size and shape of the ablation zone, but the new-generation MWA is designed to create a large predictable spherical ablation zone that is unaffected by changes in the tissue environment (12). The benefits of spherical ablation can also be superior to RFA. The elliptical spherical ablation of RFA results from the relatively narrow ablation range in the direction vertical to the electrode, such that securing of the TM may be insufficient. Spherical ablation of the new-generation MWA is considered to be likely to achieve sufficient and even TM, and the decreased number of sessions and shorter treatment times should be very therapeutically beneficial for the patient.

We developed a protocol to gradually increase the output to prevent popping based on Covidien's basic experiments, but this protocol will be improved based on future clinical experience; nevertheless, satisfactory results have been obtained that are comparable to those of RFA in this study. In the comparison between the two thermal ablation methods used in this study, there was no difference in the local recurrence rates. In patients who obtained complete necrosis with TM, there was no local recurrence in MWA, which may be because tissue ablation was obtained more reliably than in RFA due to the lower susceptibility to the heat sink effect.

Unfortunately, in this study, six patients experienced complications due to MWA. There was concern about the risk of bile duct injury due to the higher temperature and bleeding caused by the thicker antennas, but there were no significant differences in complications compared with those resulting from MWA.

There are some limitations to this study. This retrospective cohort study has not been randomized. Although there were very few differences in the clinical data between the two groups, the RFA procedure was performed early and the MWA procedure was performed later in the study, which may have resulted in bias. For $\mathrm{HCC}<3.0 \mathrm{~cm}$ in diameter located outside the hilar region, the new-generation MWA may be recommended over RFA due to its short treatment time. However, in our daily treatment protocol, HCC located near the hilar region is treated with c-TACE and percutaneous ethanol injection (PEI), so this study does not provide treatment efficacy and safety comparison between MWA and RFA. The risk of PLT complications for HCC increases by their perivascular presence. The possibility of bile duct 
injury increases especially when the mass was adjacent to the hilar region. Heat-based thermal ablation methods can also cause vascular thrombosis. Extensive thrombosis can cause liver failure in patients with poor hepatic reserves, so it must be paid a great careful to heat-based thermal ablation of the hilar region (24). The sample size may be small to detect statistically significant differences in some treatment outcomes. However, this preliminary results of early reporting on the performance of the new-generation MWA device create the basis for prospective study on this topic with less literature.

There is less experience with the new-generation MWA, and the treatment strategy is not sufficiently developed. Further accumulation of cases is necessary for a detailed comparison with RFA.

\section{Acknowledgements}

Professor Tomoki Kitawaki; Department of Mathematics, Kansai Medical University, for supporting statistical analysis.

\section{Funding}

No funding was received.

\section{Availability of data and materials}

The datasets used and/or analyzed during the present study are available from the corresponding author on reasonable request.

\section{Authors' contributions}

TS and RT designed the study and wrote the initial draft of the manuscript. TY contributed to analysis and interpretation of data and assisted in the preparation of the manuscript. KS, MY, MM, AN and YO contributed to data collection and interpretation, and critically reviewed the manuscript. All authors approved the final version of the manuscript and agree to be accountable for all aspects of the work in ensuring that questions related to the accuracy or integrity of any part of the work are appropriately investigated and resolved.

\section{Ethics approval and consent to participate}

The study protocol was conducted with the approval (approval no. 2018143) of the Ethics Committee of Kansai Medical University Medical Center (Moriguchi, Japan). All procedures performed in studies involving human participants were in accordance with the ethical standards of Clinical Research Board of Kansai Medical University Medical Center and with the 1964 Helsinki Declaration and its later amendments or comparable ethical standards. Informed consent was obtained from all individual participants included in the present study.

\section{Patient consent for publication}

Not applicable.

\section{Competing interests}

The authors declare that they have no competing interests.

\section{References}

1. El-Serag HB: Epidemiology of viral hepatitis and hepatocellular carcinoma. Gastroenterology 142: 1264-1273, 2012.

2. Lencioni R: Loco-Regional treatment of hepatocellular carcinoma in the era of molecular targeted therapies. Oncology 78: $107-112,2010$

3. Llovet JM: Updated treatment approach to hepatocellular carcinoma. J Gastroenterol 40: 225-235, 2005.

4. European Association for the Study of the Liver; European Organisation for Research and Treatment of Cancer: EASL-EORTC clinical practice guidelines: Management of hepatocellular carcinoma. J Hepatol 56: 908-943, 2012.

5. Bruix J and Sherman M; Practice Guidelines Committee, American Association for the Study of Liver Diseases: Management of hepatocellular carcinoma. Hepatology 42: 1208-1236, 2005.

6. Bruix J and Sherman M; American Association for the Study of Liver Diseases: Management of hepatocellular carcinoma: An update. Hepatology 53: 1020-1022, 2011.

7. McCarley JR and Soulen MC: Percutaneous ablation of hepatic tumors. Semin Intervent Radiol 27: 255-260, 2010.

8. Shiina S, Teratani T, Obi S, Hamamura K, Koike Y and Omata M: Nonsurgical treatment of hepatocellular carcinoma: From percutaneous ethanol injection therapy and percutaneous microwave coagulation therapy to radiofrequency ablation. Oncology 62 : 64-68, 2002

9. Yamasaki T, Kurokawa F, Shirahashi H, Kusano N, Hironaka K and Okita K: Percutaneous radiofrequency ablation therapy with combined angiography and computed tomography assistance for patients with hepatocellular carcinoma. Cancer 91: 1342-1348, 2001.

10. Lu DS, Raman SS, Limanond P, Aziz D, Economou J, Busuttil R and Sayre J: Influence of large peritumoral vessels on outcome of radiofrequency ablation of liver tumors. J Vasc Inter Radiol 14: 1267-1274, 2003.

11. Ierardi AM, Mangano A, Floridi C, Dionigi G, Biondi A, Duka E, Lucchina N, Lianos GD and Carrafiello G: A new system of microwave ablation at $2450 \mathrm{MHz}$ : Preliminary experience. Updates Surg 67: 39-45, 2015.

12. Alonzo M, Bos A, Bennett S and Ferral H: The emprint ablation system with thermosphere technology: One of the newer next-generation microwave ablation technologies. Semin Intervent Radiol 32: 335-338, 2015.

13. Thandassery RB, Goenka U and Goenka MK: Role of local ablative therapy for hepatocellular carcinoma. J Clin Exp Hepatol 4 (Suppl 3): S104-S111, 2014

14. Shiina S, Sato K, Tateishi R, Shimizu M, Ohama H, Hatanaka T, Takawa M, Nagamatsu H and Imai Y: Percutaneous ablation for hepatocellular carcinoma: Comparison of various ablation techniques and surgery. Can J Gastroenterol Hepatol 2018: 4756147, 2018.

15. Seki T, Wakabayashi M, Nakagawa T, Itho T, Shiro T, Kunieda K, Sato M, Uchiyama S and Inoue K: Ultrasonically guided percutaneous microwave coagulation therapy for small hepatocellular carcinoma. Cancer 74: 817-825, 1994.

16. Seki T, Tamai T, Nakagawa T, Imamura M, Nishimura A, Yamashiki N, Ikeda K and Inoue K: Combination therapy with transcatheter arterial chemoembolization and percutaneous microwave coagulation therapy for hepatocellular carcinoma. Cancer 89: 1245-1251,2000.

17. Vogl TJ, Farshid P, Naguib NN, Zangos S, Bodelle B, Paul J, Mbalisike EC, Beeres M and Nour-Eldin NE: Ablation therapy of hepatocellular carcinoma: A comparative study between radiofrequency and microwave ablation. Abdom Imaging 40: $1829-1837,2015$.

18. Facciorusso A, Di Maso M and Muscatiello N: Microwave ablation versus radiofrequency ablation for the treatment of hepatocellular carcinoma: A systematic review and meta-analysis. Int J Hyperthermia 32: 339-344, 2016.

19. Zhou Y, Zhao Y, Li B, Xu D, Yin Z, Xie F and Yang J: Meta-analysis of radiofrequency ablation versus hepatic resection for small hepatocellular carcinoma. BMC Gastroenterol 10: $78,2010$. 
20. Feng K, Yan J, Li X, Xia F, Ma K, Wang S, Bie P and Dong J: A randomized controlled trial of radiofrequency ablaion and surgical resection in the treatment of small hepatocellular carcinoma. J Hepatol 57: 794-802, 2012.

21. Lucchina N, Tsetis D, Ierardi AM, Giorlando F, Macchi E, Kehagias E, Duka E, Fontana F, Livraghi L and Carrafiello G: Current role of microwave ablation in the treatment of small hepatocellular carcinomas. Ann Gastroenterol 29: 460-465, 2016.

22. Dou JP, Yu J, Yang XH, Cheng ZG, Han ZY, Liu FY, Yu XL and Liang P: Outcomes of microwave ablation for hepatocellular carcinoma adjacent to large vessels: A propensity score analysis. Oncotarget 8: 28758-28768, 2017.
23. Lubner MG, Brace CL, Hinshaw JL and Lee FT Jr: Microwave tumor ablation: Mechanism of action, clinical results, and devices. J Vasc Inter Radiol 21 (8 Suppl): S192-S203, 2010.

24. Rhim H: Complications of radiofrequency ablation in hepatocellular carcinoma. Abdom Imaging 30: 409-418, 2005.

(i) (2) This work is licensed under a Creative Commons Attribution-NonCommercial-NoDerivatives 4.0 International (CC BY-NC-ND 4.0) License. 